\title{
Accurate Topological Measures for Rough Sets
}

\author{
A. S. Salama \\ Department of Mathematics, Faculty of Science, \\ Tanta University, \\ Tanta, Egypt \\ Department of Mathematics, Faculty of Science and Humanities \\ Shaqra University \\ Al-Dawadmi, KSA
}

\begin{abstract}
Data granulation is considered a good tool of decision making in various types of real life applications. The basic ideas of data granulation have appeared in many fields, such as interval analysis, quantization, rough set theory, Dempster-Shafer theory of belief functions, divide and conquer, cluster analysis, machine learning, databases, information retrieval, and many others. Some new topological tools for data granulation using rough set approximations are initiated. Moreover, some topological measures of data granulation in topological information systems are defined. Topological generalizations using $\delta \beta$-open sets and their applications of information granulation are developed.
\end{abstract}

Keywords-component; Knowledge Granulation; Topological Spaces; Rough Sets; Rough Approximations; Data Mining; Decision Making

\section{INTRODUCTION}

Granulation of the universe involves the decomposition of the universe into parts. In other words, the grouping individual elements or objects into classes, based on offering information and knowledge [7, 14,15, 21, 36,37, 42-45]. Elements in a granule are pinched together by indiscernibility, similarity, proximity or functionality [43]. The starting point of the theory of rough sets is the indiscernibility of objects or elements in a universe of concern $[14,15,17-20,51,52,21$ 22].

The original rough set theory was based on an equivalent relation on a finite universe $U$. For practical use, there have been some extensions on it. One extension is to replace the equivalent relation by an arbitrary binary relation; the other direction is to study rough set via topological method $[8,14]$. In this work, we construct topology for a family covering rough sets.

In [40] addressed four operators on a knowledge base, which are sufficient for generating new knowledge structures. Also, they addressed an axiomatic definition of knowledge granulation in knowledge bases.

Rough set theory, proposed by Pawlak in the early 1980s $[18,51-52]$, is an expansion of set theory for the study of intelligent systems characterized by inexact, uncertain or insufficient information. Moreover, this theory may serve as a new mathematical tool to soft computing besides fuzzy set theory [42-45] and has been successfully applied in machine learning, information sciences, expert systems, data reduction, and so on [28-33,34, 1-13]. In recent times, lots of researchers are interested to generalize this theory in many fields of applications [1-10].

In Pawlak's novel rough set theory, partition or equivalence (indiscernibility) relation is an important and primeval concept. But, partition or equivalence relation is still limiting for many applications. To study this matter, several interesting and having an important effect generalization to equivalence relation have been proposed in the past, such as tolerance relations, similarity relations [51], topological bases and subbases [52, 2,6] and others [4,5,11]. Particularly, some researchers have used coverings of the universe of discourse for establishing the generalized rough sets by coverings [1114]. Others [24-26,27-33] combined fuzzy sets with rough sets in a successful way by defining rough fuzzy sets and fuzzy rough sets. Furthermore, another group has characterized a measure of the roughness of a fuzzy set making use of the concept of rough fuzzy sets [34-38]. They also suggested some possible real world applications of these measures in pattern recognition and image analysis problems [24,41-46].

Topological notions like semi-open, pre-open, $\beta$ - open sets are as basic to mathematicians of today as sets and functions were to those of last century [48-52]. Then, we think the topological structure will be so important base for knowledge extraction and processing.

The topology induced by binary relations on the universes of information systems is used to generalize the basic rough set concepts. The suggested topological operations and structure open up the way for applying affluent more of topological facts and methods in the process of granular computing. In particular, the notion of topological membership function is introduced that integrates the concept of rough and fuzzy sets [17-20].

In this paper, we indicated some topological tools for data granulation by using new topological tools for rough set approximations. Moreover, we introduced using general binary relations a refinement data granulation instead of the classical equivalence relations. Section 1 gives a brief overview of data granulation structures in the universe using equivalence and general relations. Fundamentals of rough set theory under general binary relations are the main purpose of Section 2. Section 3 studies the topological data granulation properties of topological information systems. Explanation of topological data granulation in information systems appears in 
Section 4. In Section 5 we are given some more accurate topological tools for data granulation using $\delta \beta$ - open sets approach. The conclusions of our work are presented in Section 6.

\section{ESSENTIALS OF ROUGH SET APPROXIMATIONS UNDER GENERAL BINARY RELATIONS}

In rough set theory, it is usually assumed that the knowledge about objects is restricted by some indiscernibility relations. The Indiscernibility relation is an equivalence relation which is interpreted so that two objects are equivalent if we can't distinguish them using our information. This means that the objects of the given universe $U$ indiscernible by $R$ into three classes with respect to any subset $X \subseteq U$ :

Class 1: the objects which surely belong to $X$,

Class 2: the objects which possibly belong to $X$,

Class 3: the objects which surely not belong to $X$,

The object in Class 1 form the lower approximation of $X$ , and the objects of Class 1 and 3 form together its upper approximation. The boundary of $X$ consists of objects in Class 3. Some subsets of $U$ are identical to both of them approximations and they are called crisp or exact; otherwise, the set is called rough.

For any approximation space $A=(U, R)$, where $R$ is an equivalence relation, lower and upper approximations of a subset $X \subseteq U$, namely $\underline{R}(X)$ and $\bar{R}(X)$ are defined as follows:

$$
\begin{aligned}
& \underline{R}(X)=\left\{x \in U:[x]_{R} \subset X\right\}, \\
& \bar{R}(X)=\left\{x \in U:[x]_{R} \cap X \neq \phi\right\} .
\end{aligned}
$$

The lower and upper approximations have the following properties:

For every $X, Y \subset U$ from the approximation space $A=(U, R)$ we have:

$$
\begin{aligned}
& \text { 1. } \underline{R}(X) \subseteq X \subseteq \bar{R}(X), \\
& \text { 2. } \underline{R}(U)=\bar{R}(U)=U, \\
& \text { 3. } \underline{R}(\phi)=\bar{R}(\phi)=\phi, \\
& \text { 4. } \bar{R}(X \cup Y)=\bar{R}(X) \cup \bar{R}(Y), \\
& \text { 5. } \underline{R}(X \cup Y) \supseteq \underline{R}(X) \cup \underline{R}(Y), \\
& \text { 6. } \bar{R}(X \cap Y) \subseteq \bar{R}(X) \cap \bar{R}(Y),
\end{aligned}
$$

7. $\underline{R}(X \cap Y)=\underline{R}(X) \cap R(Y)$,

8. $\bar{R}(-X)=-\underline{R}(X)$,

9. $\underline{R}(-X)=-\bar{R}(X)$,

10. $\bar{R}(\bar{R}(X))=\underline{R}(\bar{R}(X))=\bar{R}(X)$,

11. $\underline{R}(\underline{R}(X))=\bar{R}(\underline{R}(X))=\underline{R}(X)$,

12. If $X \subseteq Y$, then $\bar{R}(X) \subseteq \bar{R}(Y)$ and $\underline{R}(X) \subseteq \underline{R}(Y)$.

The equality in all properties happens when $\underline{R}(X)=\bar{R}(X)=X$. The proof of all these properties can be found in [17-23,51].

Furthermore, for a subset $X \subseteq U$, a rough membership function is defined as follows: $\mu_{X}(x)=\frac{\left|[x]_{R} \cap X\right|}{\left|[x]_{R}\right|}$, where $|X|$ denotes the cardinality of the set $X$. The rough membership value $\mu_{X}(x)$ may be interpreted as the conditional probability that an arbitrary element belongs to $X$ given that the element belongs to $[x]_{R}$.

Based on the lower and upper approximations, the universe $U$ can be divided into three disjoint regions, the positive $\operatorname{POS}(X)$, the negative $N E G(X)$ and the boundary $B N D(X)$, where:

$$
\begin{aligned}
& \operatorname{POS}(X)=\underline{R}(X) \\
& \operatorname{NEG}(X)=U-\bar{R}(X) \\
& \operatorname{BND}(X)=\bar{R}(X)-\underline{R}(X)
\end{aligned}
$$

Considering general binary relations in $[18,52]$ is an extension to the classical lower and upper approximations of any subset $X$ of $U . \beta=\left\{R_{x}: x \in X\right\}$ is the base generated by the general relation defined in $[17,52]$. The general forms based on $\beta$ are defined as follows:

$$
\begin{aligned}
& \underline{R}_{\beta}(X)=\bigcup\left\{B: B \in \beta_{x}, B \subset X\right\}, \\
& \bar{R}_{\beta}(X)=\bigcup\left\{B: B \in \beta_{x}, B \cap X \neq \phi\right\}, \text { where }
\end{aligned}
$$$$
\beta_{x}=\{B \in \beta: x \in B\} \text {. }
$$

For data granulation by any binary relation, in [E. Lashein (2005) ] a rough membership function is defined as follows:

$\mu_{X}(x)=\frac{\left|X \cap\left(\cap \beta_{x}\right)\right|}{\left|\cap \beta_{x}\right|}$. 


\section{Rough SETS OF EQUIVALENCE AND GENERAL BINARY RELATIONS}

Indiscernibility as defined by equivalence relation represents a very restricted type of relationships between elements and universes. The procedure to granule the universe by general binary relations is introduced in [6].

A topological space $[1,2]$ is a pair $(X, \tau)$ consisting of a set $X$ and a family $\tau$ of subset of $X$ satisfying the following conditions:

(1) $\phi, X \in \tau$,

(2) $\tau$ is closed under arbitrary union,

(3) $\tau$ is closed under finite intersection.

The pair $(X, \tau)$ is called a topological space. The elements of $X$ are called points. The subsets of $X$ belonging to $\tau$ are called open sets. The complement of the open subsets are called closed sets. The family $\tau$ of all open subsets of $X$ is also called a topology for $X$. $\operatorname{cl}(A)=\bigcap\{F \subseteq X: A \subseteq F$ and $F$ is closed $\}$ is called $\tau$-closure of a subset $A \subset X$.

Obviously, $\operatorname{cl}(A)$ is the smallest closed subset of $X$ which contains $A$. Note that $A$ is closed iff $A=\operatorname{cl}(A)$. $\operatorname{int}(A)=\bigcup\{G \subseteq X: G \subseteq A$ and $G$ is open $\}$ is called the $\tau$-interior of a subset $A \subseteq X$. Manifestly, $\operatorname{int}(A)$ is the union of all open subsets of $X$ which contained in $A$. Make a note of that $A$ is open iff $A=\operatorname{int}(A) . b(A)=\operatorname{cl}(A)-\operatorname{int}(A)$ is called the $\tau$ boundary of a subset $A \subseteq X$.

For any subset $A$ of the topological space $(X, \tau)$, $c l(A), \operatorname{int}(A)$ and $b(A)$ are closure, interior, and boundary of $A$ respectively. The subset $A$ is exact if $b(A)=\phi$, otherwise $A$ is rough. It is clear that $A$ is exact iff $c l(A)=\operatorname{int}(A)$. In Pawlak space a subset $A \subseteq X$ has two possibilities either rough or exact.

In later years a number of generalizations of open sets have been considered [21-23]. We talk about some of these generalizations concepts in the following definitions.

Let $U$ be a finite universe set and $R$ is any binary relation defined on $U$, and $r R(x)$ be the set of all elements which are in relation to certain elements $x$ in $U$ from right for all $x \in U \quad, \quad$ in symbols $\quad r R(x)=\{x R, x \in U\} \quad$ where $x R=\{y:(x, y) \in R ; x, y \in U\}$.

Let $\beta$ be the general knowledge base (topological base) using all possible intersections of the members of $r R(x)$. The component that will be equal to any union of some members of $\beta$ must be misplaced.

\section{TOPOLOGICAL GENERALIZATIONS OF ROUGH SETS}

Let $A=(U, R)$ be an approximation space where $R$ is any binary relation defined on $U$. Then we can define two new approximations as follows:

$$
\begin{aligned}
& \underline{\tau}_{\beta}(X)=X \cap \underline{R}_{\beta}\left(\bar{R}_{\beta}(X)\right), \\
& \bar{\tau}_{\beta}(X)=X \cup \bar{R}_{\beta}\left(\underline{R}_{\beta}(X)\right) .
\end{aligned}
$$

The topological lower and the topological upper approximations have the following properties:

For every $X, Y \subset U$ and every approximation space $A=(U, R)$ we have:

1. $\underline{\tau}_{\beta}(X) \subseteq X \subseteq \bar{\tau}_{\beta}(X)$,

2. $\underline{\tau}_{\beta}(U)=U=\bar{\tau}_{\beta}(U)$,

3. $\bar{\tau}_{\beta}(\phi)=\underline{\tau}_{\beta}(\phi)=\phi$,

4. $\bar{\tau}_{\beta}(X \cup Y) \supset \bar{\tau}_{\beta}(X) \cup \bar{\tau}_{\beta}(Y)$,

5. $\underline{\tau}_{\beta}(X \cup Y) \supset \underline{\tau}_{\beta}(X) \cup \underline{\tau}_{\beta}(Y)$,

6. $\bar{\tau}_{\beta}(X \cap Y) \subset \bar{\tau}_{\beta}(X) \cap \bar{\tau}_{\beta}(Y)$,

7. $\underline{\tau}_{\beta}(X \cap Y) \subseteq \underline{\tau}_{\beta}(X) \cap \underline{\tau}_{\beta}(Y)$,

8. $\bar{\tau}_{\beta}(-X)=-\bar{\tau}_{\beta}(X)$,

9. $\underline{\tau}_{\beta}(-X)=-\underline{\tau}_{\beta}(X)$,

10. $\bar{\tau}_{\beta}\left(\bar{\tau}_{\beta}(X)\right)=\bar{\tau}_{\beta}(X)$,

11. $\underline{\tau}_{\beta}\left(\underline{\tau}_{\beta}(X)\right)=\underline{\tau}_{\beta}(X)$,

12.

If $X \subseteq Y$, then $\bar{\tau}_{\beta}(X) \subseteq \bar{\tau}_{\beta}(Y)$ and $\underline{\tau}_{\beta}(X) \subseteq \underline{\tau}_{\beta}(Y)$.

Given that topological lower and topological upper approximations satisfy that: $\underline{R}_{\beta}(X) \subseteq \underline{\tau}_{\beta}(X) \subseteq X \subseteq \bar{\tau}_{\beta}(X) \subseteq \bar{R}_{\beta}(X) \subseteq U$ this enables us to divide the universe $U$ into five disjoint regions (granules) as follows: (See Figure 1)

$$
\begin{array}{ll}
\text { 1. } & \operatorname{POS}_{\beta}(X)=\underline{R}_{\beta}(X), \\
\text { 2. } & \tau-\operatorname{POS}(X)=\underline{\tau}_{\beta}(X)-\underline{R}_{\beta}(X), \\
\text { 3. } & \tau-B N D(X)=\bar{\tau}_{\beta}(X)-\underline{\tau}_{\beta}(X), \\
\text { 4. } & \tau-N E G(X)=\bar{R}_{\beta}(X)-\bar{\tau}_{\beta}(X), \\
\text { 5. } & N E G_{\beta}(X)=U-\bar{R}_{\beta}(X) .
\end{array}
$$


The following theorems study the properties and relationships among the above regions namely boundary, positive and negative regions.

Theorem 4.1 let $I S=\left(U, A, \tau_{R}\right)$ be a topological information system and for any subset $X \subset U$ we have:

(1) $\tau-B N D(X) \cap \underline{\tau}_{\beta}(X)=\phi$,

(2) $\tau-B N D(X) \cap \tau-N E G(X)=\phi$,

(3) $\bar{\tau}_{\beta}(X)=\underline{\tau}_{\beta}(X) \cup \tau-B N D(X)$,

(4) $\underline{\tau}_{\beta}(X), \tau-N E G(X)$ and $\tau-B N D(X)$ are disjoint granules of $U$.

Proof: You can make use of Figure 1.

Theorem 4.2 let $I S=\left(U, A, \tau_{R}\right)$ be a topological information system and for any subsets $X, Y \subset U$ we have:

(1) $\tau-B N D(U)=\phi$,

(2) $\tau-B N D(X)=\tau-B N D(U-X)$,

(3) $\tau-B N D(\tau-B N D(X)) \subset \tau-B N D(X)$, $\tau-B N D(X \cap Y) \subset \tau-B N D(X) \cup \tau-B N D(Y)$

Proof: (1) and (2) is obvious, by definitions.

$$
\tau-B N D(\tau-B N D(X))
$$

$=\bar{\tau}_{\beta}\left(\bar{\tau}_{\beta}(X) \cap \bar{\tau}_{\beta}(U-X)\right)$

$$
=\tau-B N D\left(\bar{\tau}_{\beta}(X) \cap \bar{\tau}_{\beta}(U-X)\right)
$$

$\cap \bar{\tau}_{\beta}\left(U-\left(\bar{\tau}_{\beta}(X) \cap \bar{\tau}_{\beta}(U-X)\right)\right)$

$\subset \bar{\tau}_{\beta}(X) \cap \bar{\tau}_{\beta}(U-X)=\tau-B N D(X)$.

(4)

$\tau-B N D(X \cap Y)=\bar{\tau}_{\beta}(X \cap Y) \cap \bar{\tau}_{\beta}(U-X \cap Y)$

Theorem 4.3 let $I S=\left(U, A, \tau_{R}\right)$ be a topological information system and for any subset $X, Y \subset U$ we have:

(1) $U=\tau-N E G(\phi)$,

(2) $\tau-N E G(X)=\underline{\tau}_{\beta}(U-X)$,

(3) $X \cap \tau-N E G(X)=\phi$,

(4) $\tau-N E G(U-\tau-N E G(X))=\tau-N E G(X)$, $\tau-N E G(X \cup Y)$

$\subset \tau-N E G(X) \cup \tau-N E G(Y)$,

$\tau-N E G(X \cap Y)$

$\supset \tau-N E G(X) \cap \tau-N E G(Y)$

Proof: (1), (2), (3) and (4) are obvious.

(5)

$$
\begin{aligned}
& \tau-\operatorname{NEG}(X \cup Y) \\
& =U-\bar{\tau}_{\beta}(X \cup Y) \subset U-\left(\bar{\tau}_{\beta}(X) \cup \bar{\tau}_{\beta}(Y)\right)
\end{aligned}
$$

$$
\begin{aligned}
= & \left(U-\bar{\tau}_{\beta}(X)\right) \cap\left(U-\bar{\tau}_{\beta}(Y)\right) \\
& \subset \tau-\operatorname{NEG}(X) \cup \tau-\operatorname{NEG}(Y) \\
& \quad \tau-\operatorname{NEG}(X) \cap \tau-\operatorname{NEG}(Y) \\
& =\left(U-\bar{\tau}_{\beta}(X)\right) \cap\left(U-\bar{\tau}_{\beta}(Y)\right) \\
= & U-\left(\bar{\tau}_{\beta}(X) \cup \bar{\tau}_{\beta}(Y)\right) \subset U-\bar{\tau}_{\beta}(X \cap Y) \\
= & \tau-\operatorname{NEG}(X \cap Y)
\end{aligned}
$$

Example 4.1 let $U=\left\{u_{1}, u_{2}, u_{3}, u_{4}, u_{5}, u_{6}, u_{7}\right\}$ be the universe of 7 patients have data sheets shown in Table I with possible dengue symptoms. If some experts give us the general relation $R$ defined among those patients as follows:

TABLE I. PATIENTS INFORMATION SYSTEM

\begin{tabular}{|l|l|l|l|l|}
\hline \multirow{2}{*}{$U$} & \multicolumn{3}{|l|}{ Conditional Attributes (C) } & $\begin{array}{l}\text { Decision } \\
\text { (D) }\end{array}$ \\
\cline { 2 - 5 } & Temperature & Flu & Headache & Dengue \\
\hline u1 & Normal & No & No & No \\
\hline u2 & High & No & No & No \\
\hline u3 & Very High & No & No & Yes \\
\hline u4 & High & No & Yes & Yes \\
\hline u5 & Very High & No & Yes & Yes \\
\hline u6 & High & Yes & Yes & Yes \\
\hline u7 & Very High & Yes & Yes & Yes \\
\hline
\end{tabular}

$R=\left\{\left(u_{1}, u_{1}\right),\left(u_{1}, u_{7}\right),\left(u_{2}, u_{2}\right),\left(u_{3}, u_{3}\right)\right.$,

$\left(u_{3}, u_{6}\right),\left(u_{4}, u_{4}\right),\left(u_{5}, u_{5}\right),\left(u_{6}, u_{6}\right)$

,$\left.\left(u_{7}, u_{7}\right)\right\}$. form:

The topological knowledge base will take the following

$$
\beta=\left\{\left\{u_{1}, u_{7}\right\},\left\{u_{2}\right\},\left\{u_{3}, u_{6}\right\},\left\{u_{4}\right\},\left\{u_{5}\right\},\left\{u_{6}\right\},\left\{u_{7}\right\}\right\}
$$

For some patients $X=\left\{u_{2}, u_{3}, u_{7}\right\}$ the upper and lower approximations based on the topological knowledge base are given by:

$$
\bar{R}_{\beta}(X)=\left\{u_{1}, u_{2}, u_{3}, u_{6}, u_{7}\right\} \text {, and } \quad \underline{R}_{\beta}=\left\{u_{2}, u_{7}\right\} .
$$

By using the lower and upper approximations, the granules of universe are three disjoint regions as follows:

$$
\begin{gathered}
\operatorname{POS}_{\beta}(X)=\underline{R}_{\beta}(X)=\left\{u_{2}, u_{7}\right\}, \\
B N D_{\beta}(X)=\bar{R}_{\beta}(X)-\underline{R}_{\beta}(X)=\left\{u_{1}, u_{3}, u_{6}\right\}, \\
N E G_{\beta}(X)=U-\bar{R}_{\beta}(X)=\left\{u_{4}, u_{5}\right\} .
\end{gathered}
$$

According to the topological knowledge base we can easily see that:

$\bar{\tau}_{\beta}(X)=\left\{u_{1}, u_{2}, u_{3}, u_{7}\right\}, \underline{\tau}_{\beta}(X)=\left\{u_{2}, u_{3}, u_{7}\right\}$.

Then we have the following granules of the universe:

$$
\text { 1. } \operatorname{POS}_{\beta}(X)=\{u 2, u 7\} \text {, }
$$




$$
\begin{array}{ll}
\text { 2. } & \tau-\operatorname{POS}(X)=\{u 3\}, \\
\text { 3. } & \tau-B N D(X)=\{u 1\}, \\
\text { 4. } & \tau-N E G(X)=\{u 6\} \\
\text { 5. } & N E G_{\beta}(X)=\{u 4, u 5\} .
\end{array}
$$

\section{New Topological Generalizations of Rough Sets}

In this section, we used the topological tool $\delta \beta$-open sets to introduce the concepts of $\delta \beta$-lower and $\delta \beta$-upper approximations. The suggested model helps in decreasing the boundary region of concepts in information systems. Also, we use the topological measure $\alpha_{R_{\delta \beta}}$ is used as a topological accurate measure of data granulation correctness.

For any subset $X$ of a topological space $(U, \tau)$. The $\delta$ closure of a subset $X$ is defined by $c_{\delta}(X)=\{x \in U: X \cap \operatorname{int}(\operatorname{cl}(G)) \neq \phi, G \in \tau$

and $x \in G\}$ A set $X$ is called $\delta$-closed if $X=\mathrm{cl}_{\delta}(X)$. The complement of a $\delta$-closed set is called $\delta$-open.

$$
\text { Notice that } \operatorname{int}_{\delta}(X)=U \backslash c l_{\delta}(U \backslash X) \text {. }
$$

A subset $X$ of a topological space $(U, \tau)$ is called $\delta \beta$ open if $X \subseteq \operatorname{cl}\left(\operatorname{int}\left(c l_{\delta}(X)\right)\right)$.

Let $(U, \tau)$ be a topological space and $X \subseteq U$, the following new topological tools of any subset $X$ are defined as follows $[1,2,6]$ :

- Regular open tool if $X=\operatorname{Int}(C l(X))$.

- Semi-open tool if $X \subset C l(\operatorname{Int}(X))$.

- $\alpha$-open tool if $X \subset \operatorname{Int}(C l(\operatorname{Int}(X)))$.

- Pre-open tool if $X \subset \operatorname{Int}(C l(X))$.

- Semi pre open tool $(\beta-$ open $)$ if $X \subset C l(\operatorname{Int}(C l(X)))$.

The family of all $\delta \beta$-open sets of $U$ is denoted by $\delta \beta O(U)$. The complement of $\delta \beta$-open set is called $\delta \beta$ closed set. The family of $\delta \beta$-closed sets are denoted by $\delta \beta C(U)$.

Let $X$ be a subset of a topological space $(U, \tau)$, then we have: (i) The union of all $\delta \beta$-open sets contained inside $X$ is called the $\delta \beta$-interior of $X$ and is denoted by $\beta$ int ${ }_{\delta}(X)$.

(ii) The intersection of all $\delta \beta$-closed sets containing $X$ is called the $\delta \beta$-closure of $X$ and is denoted by $\beta c l,(X)$.

Lemma 6.1 For a subset $X$ of a topological space $(U, \tau)$ we have:

(i) $\operatorname{\beta int}_{\delta}(X)=X \cap \operatorname{cl}(\operatorname{int}(\operatorname{cl}(X)))$.

(ii) $\beta c{ }_{\delta}(X)=X \cup \operatorname{int}(\operatorname{cl}(\operatorname{int}(X)))$.

$\delta \beta$-open sets is stronger than any topological near open sets such as $\delta$-open, regular open, semi-open, $\alpha-$ open, pre-open, $\beta$-open.

The following example illustrates the above note.

Example 5.1 Let $(U, \tau)$ be a topological space where, $U=\{a, b, c, d, e\}$ and $\tau=\{U, \varphi,\{d\},\{e\},\{a, d\},\{d, e\}$, $\{a, d, e\},\{b, c, e\},\{b, c, d, e\}\}$ $\{a, c\} \in \delta \beta O(U) \quad$ but $\quad\{a, c\} \notin \delta O(U)$ $\{b, d, e\} \in \delta \beta O(U) \quad$ but $\quad\{b, d, e\} \notin R O(U)$, $\{a, e\} \in \delta \beta O(U)$ but $\{a, e\} \notin P O(U),\{c\} \in \delta \beta O(U)$ but $\{c\} \notin \beta O(U),\{b\} \in \delta \beta O(U)$ but $\{b\} \notin S O(U)$ and $\{c, d\} \in \delta \beta O(U)$ but $\{c, d\} \notin \alpha O(U)$. Where $\delta O(U), R O(U), S O(U), \alpha O(U), P O(U)$ and $\beta O(U)$ denoted the family of all $\delta$-open, regular open, semi-open, $\alpha$-open, pre-open and $\beta$-open sets of $U$ respectively.

Arbitrary union of $\delta \beta$-open sets is again $\delta \beta$-open set, but the intersection of two $\delta \beta$-open sets may not be $\delta \beta$ open set. Thus the $\delta \beta$-open sets in a space $U$ do not form a topology.

Let $U$ be a finite non-empty universe. The pair $\left(U, R_{\delta \beta}\right)$ is called a $\delta \beta$-approximation space where $R_{\delta \beta}$ is a general relation used to get a subbase for a topology $\tau$ on $U$ which generates the class $\delta \beta O(U)$ of all $\delta \beta$-open sets.

Example 6.2 Let $U=\{a, b, c, d, e\}$ be a universe and a relation $R$ defined by $R=\{(a, a),(a, e),(b, c),(b, d)$, $(c, e),(d, a),(d, e),(e, e)\}$,thus $a R=d R=\{a, e\}$, 
$b R=\{c, d\}$ and $c R=e R=\{e\}$. Then the topology associated with this relation is $\tau=\{U, \phi,\{e\}$, $\{a, e\},\{c, d\}, \quad\{c, d, e\}, \quad\{a, c, d, e\}\} \quad$ earned $\delta \beta O(U)=P(U)-\{b\}$. So $\left(U, R_{\delta \beta}\right)$ is a $\delta \beta$ approximation space.

Let $\left(U, R_{\delta \beta}\right)$ be a $\delta \beta$ - approximation space. $\delta \beta$-lower approximation and $\delta \beta$-upper approximation of any nonempty subset $X$ of $U$ is defined as:

$$
\begin{gathered}
\underline{R}_{\delta \beta}(X)=\bigcup\{G \in \delta \beta O(U): G \subseteq X\} \\
\bar{R}_{\delta \beta}(X)=\bigcap\{F \in \delta \beta C(U): F \supseteq X\} . \\
\quad \underline{R}(X) \subseteq \underline{R}_{\beta}(X) \subseteq \underline{R}_{\delta \beta}(X) \subseteq X \\
\text { We see that: } \bar{R}_{\delta \beta}(X) \subseteq \bar{R}_{\beta}(X) \subseteq \bar{R}(X)
\end{gathered} .
$$

Let $\left(U, R_{\delta \beta}\right)$ be a $\delta \beta$ - approximation space, $X \subseteq U$. From the relation $\operatorname{int}(X) \subseteq \beta \operatorname{int}(X) \subseteq \delta \beta \operatorname{int}(X) \subseteq X$

$$
\subseteq \delta \beta c l(X) \subseteq \beta \operatorname{cl}(X) \subseteq \operatorname{cl}(X)
$$

The Universe $U$ can be separated into divergent 24 granules with respect to any $X \subseteq U$.

We can distinguish the degree of completeness of granules of $U$ by the topological tool named $\delta \beta$-accuracy measure defined for any granule $X \subseteq U$ as follows: $\alpha_{R}(X)=\frac{\left|\underline{R}_{\delta \beta}(X)\right|}{\left|\bar{R}_{\delta \beta}(X)\right|}$ where $X \neq \phi$.

Example 5.2 According to Example 5.1 we can construct the following table (Table II) showing the degree of accuracy measure $\alpha_{R}(X), \beta$-accuracy measure $\alpha_{R_{\beta}}(X)$ and $\delta \beta$ accuracy measure $\alpha_{R_{\delta \beta}}(X)$ for some granules of $U$.

TABLE II. ACCURACY MEASURES OF SOME GRANULES

\begin{tabular}{|c|c|c|c|}
\hline $\begin{array}{c}\text { Some } \\
\text { granules }\end{array}$ & $\begin{array}{c}\text { Pawlak's } \\
\text { accuracy }\end{array}$ & $\beta$-accuracy & $\delta \beta$-accuracy \\
\hline$\{\mathrm{b}, \mathrm{d}\}$ & $0 \%$ & $100 \%$ & $100 \%$ \\
\hline$\{\mathrm{b}, \mathrm{e}\}$ & $33.3 \%$ & $66.6 \%$ & $100 \%$ \\
\hline$\{\mathrm{a}, \mathrm{b}, \mathrm{e}\}$ & $66.6 \%$ & $100 \%$ & $100 \%$ \\
\hline$\{\mathrm{a}, \mathrm{c}, \mathrm{d}\}$ & $50 \%$ & $66.6 \%$ & $100 \%$ \\
\hline$\{\mathrm{b}, \mathrm{c}, \mathrm{d}, \mathrm{e}\}$ & $60 \%$ & $80 \%$ & $100 \%$ \\
\hline
\end{tabular}

We see that the degree of accuracy of the granule $\{b, c, d, e\}$ using Pawlak's accuracy measure equal to $60 \%$, using $\beta$-accuracy measure equal to $80 \%$ and using $\delta \beta$ accuracy measure equal to $100 \%$. Accordingly $\delta \beta$ - accuracy measure is more precise than Pawlak's accuracy and $\beta$-accuracy measures.

\section{CONCLUSIONS AND APPLICATION NOTES}

In the near future is the completion of a new paper for the application of the granules concepts of this paper in medicine especially in the field of heart disease in collaboration with specialists in this field. We designed a JAVA application program novelty to generate granules division automatically once you select points covered by the heart scan and the medical relationship among them using topology defined on it. The program works under any operating system but needs to be a great RAM memory and strong processor to end the division of the millions of points to the granules in seconds.

\section{REFERENCES}

[1] H.M. Abu-Donia (2012), Multi knowledge based rough approximations and applications Knowledge-Based Systems, Volume 26, , Pages 20-29

[2] D. Andrijevic(1986) , Semi-pre, open sets, Mat. Vesnik. 38, 24-32.

[3] Tutut Herawan, Mustafa Mat Deris (2010), Jemal H. Abawajy, A rough set approach for selecting clustering attribute Knowledge-Based Systems, Volume 23, Issue 3, , Pages 220-231

[4] Jiye Liang (2009), Junhong Wang, Yuhua Qian, A new measure of uncertainty based on knowledge granulation for rough sets. Information Sciences, 179, 458-470.

[5] E. Lashein (2005) , A.M. Kozae, , A. Abo Khadra, T. Medhat, Rough Set Theory for Topological Spaces, International Journal of Approximate Reasoning 40, 35-43.

[6] T.Y. Lin (1998), Granular Computing on Binary Relations I: data mining and neighborhood systems, II: rough set representations and belief functions, In: Rough Setsin Knowledge Discovery 1, L. Polkowski, A.Skowron (Eds.), Phys.-Verlag, Heidelberg, 107-14.

[7] T.Y. Lin (2002), Y.Y. Yao, L.A. Zadeh, Data Mining, Rough Sets and Granular Computing (Studies in Fuzziness and Soft Computing), Physica-Verlag, Heidelberg.

[8] Guilong Liu (2009), Ying Sai, A comparison of two types of rough sets induced by coverings, International Journal of Approximate Reasoning 50, 521-528.

[9] Yee Leung (2008), Manfred M. Fischer, Wei-Zhi Wu , Ju-Sheng Mi, A rough set approach for the discovery of classification rules in intervalvalued information systems, International Journal of Approximate Reasoning 47, 233-246.

[10] Guilong Liu (2010), Rough set theory based on two universal sets and its applications Knowledge-Based Systems, 23(2),110-115

[11] Guilong Liu(2008), Axiomatic systems for rough sets and fuzzy rough sets, , International Journal of Approximate Reasoning 48, 857-867.

[12] A. S. Mashhour (1982), M. E. Abd El-Monsef, S. N. El-Deeb , On precontinuous and week pre-continuous mappings, Proc. Math. \& phys. Soc. Egypt 53, 47-53.

[13] T. Nishino(2005), M. Nagamachi, H. Tanaka, Variable Precision Bayesian Rough Set Model and Its Application to Human Evaluation Data, RSFDGrC 2005, LNAI 3641, Springer Verlag, 294-303.

[14] T. Nishino (2006), M.Sakawa, K. Kato, M. Nagamachi, H.Tanak, Probabilistic Rough Set Model and Its Application to Kansei Engineering, Transactions on Rough Sets V (Inter. J. of Rough Set Society), LNCS 4100, Springer, 190-206.

[15] O. Njasted, On some classes of nearly open sets, Pro. J. Math. 15 (1965) 961-970.

[16] N. Levine (1963), Semi open sets and semi continuity topological spaces, Amer. Math. Monthly 70 ,24-32.

[17] Zhi Pei (2011), Daowu Pei, Li Zheng, Topology vs generalized rough sets, International Journal of Approximate Reasoning 52, 231-239.

[18] Zhi Pei (2011), Daowu Pei, Li Zheng, Covering rough sets based on neighborhoods an approach without using neighborhoods, International Journal of Approximate Reasoning 52, 461-472. 
[19] L. Polkowski and A.Skowron (1998),Towards Adaptive Calculus of Granules, Proceedings of 1998 IEEE Inter. Conf. on Fuzzy Sys., 111116.

[20] Z. Pawlak, A. Skowron (2007), Rough sets and Boolean reasoning, Information Sciences $177,41-73$.

[21] Z. Pawlak, A. Skowron (2007), Rough sets: some extensions, Information Sciences 177, 28-40.

[22] Z. Pawlak, A. Skowron (2007), Rudiments of rough sets, Information Sciences 177, 3-27.

[23] Z. Pawlak (1981), Rough sets, Int. J. Comput. Information Sciences 11, 341-356.

[24] Yuhua Qian, Jiye Liang (2009), Chuangyin Dang, Knowledge structure, knowledge granulation and knowledge distance in a knowledge base, International Journal of Approximate Reasoning 50, 174-188.

[25] Yuhua Qian, Liang Jiye (2010), Yao Yiyu, Dang Chuangyin MGRS: A multi-granulation rough set, Information Sciences 180, 949-970.

[26] Hu Qinghua (2008), Liu Jinfu, Yu Daren, Mixed feature selection based on granulation and approximation, Knowledge-based system, 21, 294-304.

[27] A. S. Salama (2008), Topologies Induced by Relations with Applications, journal of Computer Science 4, 879-889.

[28] A. S. Salama (2008), Two New Topological Rough Operators, J. of Interdisciplinary Math. Vol. 11, No.1, New Delhi Taru Publications-, INDIA $1-10$.

[29] A. S. Salama (2010); Topological Solution for missing attribute values in incomplete information tables, Information Sciences 180, 631-639.

[30] D. Slezak (2004), The Rough Bayesian Model for Distributed Decision Systems, RSCT 2004, LNAI 3066, Springer Verlag, 384-393.

[31] D. Slezak (2005), Rough Sets and Bayes factors, Transactions on Rough Set III, LNCS 3400, 202-229.

[32] D. Slezak (2002), W.Ziarko, Bayesian Rough Set Model, In: Proc. of the Int. Workshop on Foundation of Data Mining (FDM 2002), December 9, Maebashi, Japan ,131-135.

[33] D. Slezak, W.Ziarko(2003), Variable Precision Bayesian Rough Set Model, RSFDGrC 2003, LNAI 2639, Springer Verlag, 312-315.

[34] Andrzej Skowron (1996), Jaroslaw Stepaniuk, Tolerance Approximation Spaces. Fundam. Inform. 27(2-3): 245-253

[35] Andrzej Skowron (2012), Jaroslaw Stepaniuk, Roman W. Swiniarski, Modeling rough granular computing based on approximation spaces. Information Sciences 184(1): 20-43

[36] D. J. Spiegelhalter (2004), K. R. Abrams, J. P. Myles, " Bayesian Approaches to Clinical Trials and Health-Care Evaluation". John Wiley \& Sons Ltd, The Atrium, Southern Gate, Chichester, England.

[37] D.Slezak, W.Ziarko(2003), Attribute Reduction in the Bayesian Version of Variable Precision Rough Set Model, In: Proc. of RSKD, ENTCS, 82, 4-14.

[38] D.Slezak, W.Ziarko (2005), The Investigation of the Bayesian Rough Set Model, International Journal of Approximate Reasoning vol.40, 81-91.

[39] Yanhong She (2012), Xiaoli $\mathrm{He}$, On the structure of the multigranulation rough set model Knowledge-Based Systems, In Press, Uncorrected Proof, Available online 12 June 2012
[40] You- Chen Shyang (2012), Classifying credit ratings for Asian banks using integrating feature selection and the CPDA-based rough sets approach Knowledge-Based Systems, Volume 26, , Pages 259-270

[41] Ronald R. Yager(2009), Comparing approximate reasoning and probabilistic reasoning using the Dempster-Shafer framework, International Journal of Approximate Reasoning 50, 812-821.

[42] E. A. Rady, A. M. Kozae (2004), M. M. E. Abd El-Monsef, Generalized Rough Sets, Chaos, Solitons, \& Fractals 21, 49-53.

[43] Y.Y.Yao(1998), Constructive and algebraic methods of theory of rough sets, Information Sciences 109, 21-47.

[44] Y.Y.Yao (1998), Relational interpretations of neighborhood operators and rough set approximation operators, Information Sciences 111, 239259.

[45] Y.Yang,R.I.John (2008), Generalizations of roughness bounds in rough set operations, International Journal of Approximate Reasoning 48, 868878.

[46] Y. Yao, Y. Zhao(2008); Attribute reduction in decision-theoretic rough set models. Information Sciences 178, 3356-3373.

[47] Y.Y. Yao(1999), Granular Computing using Neighborhood Systems, in: Advances in Soft Computing: Engineering Design and Manufacturing, R. Roy, T. Furuhashi, and P. K. Chawdhry (Eds.), Springer-Verlag, London, 539-553.

[48] A.M. Zahran(2000), Regularly open sets and a good extension on fuzzy topological spaces, Fuzzy Sets and Systems 116, 353-359.

[49] L.A. Zadeh(1979), "Fuzzy Sets and Information Granularity". In: Advances in Fuzzy Set Theory and Applications, Gupta, N., Ragade, R. and Yager, R. (Eds.), North- Holland, Amsterdam, 3-18.

[50] L. A. Zadeh (1997) , "Towards a Theory of Fuzzy Information Granulation and its Centrality in Human Reasoning and Fuzzy Logic". Fuzzy Sets and Systems, 19, 111-127.

[51] L. A. Zadeh (2006), Generalized theory of uncertainty (GTU) principal concepts and ideas, Computational Statistics \& Data Analysis, 51(1) $15-46$

[52] L. A. Zadeh (2002), Toward a perception-based theory of probabilistic reasoning with imprecise probabilities Journal of Statistical Planning and Inference, 105(1), 233-264

\section{AUTHORS PROFILE}

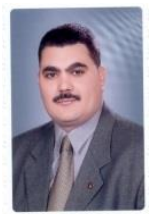

A. S. Salama received the BS degree in mathematics from Tanta University, Tanta, Egypt, in 1998 and the MS degree in Topological Rough Sets from the University Of Tanta, Egypt, in 2002. He worked at Tanta University from 1998 to 2008. He studied as a PhD student at Tanta University, Tanta, Egypt, from 2002 to 2004, in Topology and Information Systems. He was Associate Professor in the Department of Mathematics at College of Science in Dawadmi in King Saud University, KSA from 2008 until 2010. Currently, He is Associate Professor at Shaqra University, KSA. His research interests include artificial intelligence, rough set, Data mining, Topology, Fuzzy sets, Information Systems. 